\title{
HIGH PRECISION LATTICE QCD MEETS EXPERIMENT
}

\author{
P. LEPAGE \\ Laboratory for Elementary-Particle Physics, Cornell University,Ithaca, NY 14853, USA \\ E-mail: gpl@mail.lns.cornell.edu \\ C. DAVIES \\ Dept. of Physics and Astronomy, University of Glasgow, Glasgow, G12 8QQ, UK \\ E-mail: c.davies@physics.gla.ac.uk
}

\begin{abstract}
We review recent results in lattice QCD from numerical simulations that allow for a much more realistic QCD vacuum than has been possible before. Comparison with experiment for a variety of hadronic quantities gives agreement to within statistical and systematic errors of $3 \%$. We discuss the implications of this for future calculations in lattice QCD, particularly those which will provide input for $B$-factory experiments.
\end{abstract}

\section{Introduction}

In this talk we report on recent progress in lattice QCD simulations that finally allows precise calculations of a broad, but restricted, range of important non-perturbative quantities. For most of its 25 year history, high precision calculations in lattice QCD have been stymied by our inability to include realistic effects from light-quark vacuum polarization. Small quark masses, for $u$ and $d$ quarks in particular, were too expensive. Consequently quark vacuum polarization was omitted ("quenched QCD") in most work. When vacuum polarization was included, usually only $u$ and $d$ quarks were kept (no $s$ ) and these had masses 10-20× too large. Such approximations led to uncontrolled systematic errors that could be $10-30 \%$ or larger in almost all lattice QCD calculations.

During the past three years, several lattice QCD groups have been exploring a new discretization of the quark action that is $50-1000$ times faster than previous discretizations, but also highly accurate. These investigations have led to a series of simulations that include $u, d$, and $s$ quark vacuum polarization, with $u$ and $d$ masses that are $3-5 \times$ smaller than before. These masses are still unrealistically large, but they are small enough to allow accurate extrapolations to the physical masses. Consequently lattice QCD errors can be reduced to a few percent, and high precision non-perturbative QCD is now possible for the first time.

High precision non-perturbative QCD is essential to the experimental study of the Standard Model. The CKM parameters $\rho$ and $\eta$, for example, are con- strained by experimental and theoretical results for $B-\bar{B}$ mixing, $B \rightarrow \pi l \nu, K-\bar{K}$ mixing, $\ldots$. Each of these quantities has a non-perturbative QCD part and a weak interaction part. Current uncertainties, of order $20 \%$, in the QCD parts dominate the uncertainties in $\rho$ and $\eta$. It is critically important that non-perturbative QCD errors be reduced to a few percent, which is of order the experimental errors expected from $B$-factories, CLEO-c, and the hadron colliders.

High precision non-perturbative quantum field theory may well be important for beyond the Standard Model, as well. Two of the three known interactions (QCD and gravity) are strongly coupled. New strongly-coupled field theories are quite possible, even likely, at LHC energies and/or beyond. Strong coupling is generic at low energies in nonabelian gauge theories, unless the gauge symmetry is spontaneously broken; and, even then, the most likely symmetry breaking mechanisms are dynamical, which again requires strong coupling.

Here we will review the new developments in lattice QCD techniques, and discuss recent calculations, and possibilities (and limitations) for the future.

\section{Lattice QCD Calculations}

Lattice QCD calculations proceed by the discretization of a 4-d box of space-time into a lattice. The QCD Lagrangian is then discretized onto that lattice. The spacing between the points of the lattice, $a$, is $\approx 0.1 \mathrm{fm}$ in current calculations and the length of a side of the box is $L \approx 3.0 \mathrm{fm}$. Thus our simulations can cover energy scales from $\approx 2 \mathrm{GeV}$ down to $\approx 100 \mathrm{MeV}$. 
The Feynman Path Integral is evaluated numerically in a two-stage process. In the first stage, sets of gluon fields ("configurations") are created which are representative "vacuum snapshots". In the second stage, quarks are allowed to propagate on these background gluon field and hadron correlators are calculated. The dependence of the correlators on lattice time is exponential. From the exponent the masses of hadrons of a particular $J^{P C}$ can be extracted, and from the amplitude, simple matrix elements.

QCD as a theory has a number of unknown parameters, the overall dimensionful scale of QCD (三 the bare coupling constant) and the bare quark masses. To make predictions, these parameters must be fixed from experiment. In lattice QCD we do this by using one hadron mass for each parameter. The quantity which is equivalent to the overall scale of QCD on the lattice is the lattice spacing.

Lattice calculations are hard and time consuming. Progress has occurred in the last thirty years through gains in computer power but also, more importantly, through gains in calculational efficiency and physical understanding. One particular area which revolutionized the field from the mid-1980s was the understanding of the origin of discretization errors and their removal by improving the lattice QCD Lagrangian. Discretization errors appear whenever equations are discretized and solved numerically. They manifest themselves as a dependence of the physical result on the unphysical lattice spacing. In lattice QCD, as elsewhere, they are corrected by the adoption of a higher order discretization scheme. The complication in a quantum field theory like QCD is the presence of radiative corrections to the coefficients in the higher order scheme which must be determined.

Determining such radiative corrections is a major challenge for lattice QCD theorists. These involve physics at momentum scales of order $\pi / a$, where $a$ is the lattice spacing, and, therefore they can be analyzed using perturbation theory ${ }^{1}$ when $a$ is small enough, because of asymptotic freedom. The oneand two-loop perturbative analyses that are required are complicated by the exceedingly unwieldy Feynman rules of lattice QCD. The rule for a single vertex can run to several hundred pages of 6 pt text. Computer automation is essential. Progress on these calculations is currently the limiting factor in most high precision work that is relevant to tests of the
Standard Model.

Physical understanding of heavy quark physics on the lattice has also made a huge difference to the feasibility of calculating matrix elements relevant to the $B$-factory program on the lattice. The use of non-relativistic effective theories requires the lattice to handle only scales appropriate to the physics of the non-relativistic bound states and not the (large) scale associated with the $b$ quark mass. $B$ physics is now one of the areas where lattice QCD can make the most impact.

One area which has remained problematic, but which this year's results have addressed successfully, is the handling of light quarks on the lattice. In particular the problem is how to include the dynamical (sea) $u / d / s$ quark pairs that appear as a result of energy fluctuations in the vacuum. We can safely ignore $b / c / t$ quarks in the vacuum because they are so heavy, but we know that light quark pairs have significant effects, for example in screening the running of the coupling constant and in generating Zweigallowed decay modes for unstable mesons.

Because quarks are fermions, they cannot be simulated directly on the computer, but must be "integrated out" of the Feynman Path Integral. This leaves a QCD Lagrangian in terms of gluon fields which includes $\ln (\operatorname{det}(M))$ where $M$ is an enormous $\left(10^{7} \times 10^{7}\right)$ sparse matrix. The inclusion of dynamical quarks is then numerically very expensive, particularly as the quark mass is reduced towards the small values which we know the $u$ and $d$ quarks have.

Many calculations even today use the "quenched approximation" in which the light quark pairs are ignored. Results then suffer from a systematic error of $\mathcal{O}(20 \%)$. A serious problem with the quenched approximation is the lack of internal consistency which means that the results depend on the hadrons that were used to fix the parameters of QCD. This ambiguity plagues the lattice literature.

Other calculations have included 2 flavors of degenerate dynamical quarks, i.e. $u$ and $d$, but with masses $10-20 \times$ the physical ones. This approximation is better than the quenched approximation but large uncertainties remain because the $s$ quark is omitted. Results must also be extrapolated to the physical $u / d$ quark mass and chiral perturbation theory is a good tool for this. However, chiral perturbation theory only works well if the $u / d$ quark mass is light enough and, for errors at the few percent level, 
this means less than $m_{s} / 2$. This has been impossible to achieve in most calculations.

New results this year ${ }^{2}$ have included $u, d$ and $s$ quarks in the vacuum, with light enough $u / d$ masses to perform accurate chiral extrapolations. The results use a new discretization of the quark action the numerically fast improved staggered formalism. This formalism is well-matched to the supercomputing power of a few Tflops that is currently achievable.

\subsection{Improved Staggered Quarks}

The starting point for the staggered quark formalism is the naïve discretization of the Dirac quark action onto a lattice. This action has good features: chiral symmetry and discretization errors that appear only as the square and higher powers of the lattice spacing. The naïve discretization suffers from the notorious doubling problem, however. A single quark species on the lattice gives rise to 16 quark species, or tastes, on a 4-d lattice. The additional tastes appear around the edges of the Brilliouin zone, where $p \approx \pi / a$, as copies of a $p \approx 0$ quark. This would not be a problem if there were no interaction between the different tastes since the quark action would then fall apart into 16 different pieces in an appropriate basis and we could take $\operatorname{det}(M)^{(1 / 16)}$ in simulations to give the effect of 1 quark flavor.

There is interaction between the different tastes, however. It is mediated by highly virtual gluons, with momenta around $\pi / a$. A quark of one taste can absorb or emit such a high momentum gluon and turn into a quark of another taste. The effects of this taste-changing interaction are quite severe for the naïve action, giving rise to large discretization errors (even though formally of $\mathcal{O}\left(a^{2}\right)$ ) and large perturbative renormalization factors, e.g. for the quark mass, when translating from the lattice scheme to the continuum. The degeneracy in mass of mesons made from quarks of different taste is lost. This is most noticeable for the pions because there is a light Goldstone boson.

Because the taste-changing interaction is a high momentum one it can be understood in lattice perturbation theory. In particular, the effects can be significantly improved by suppressing the coupling of quarks to gluons of momenta $\pi / a$ in any direction. This is achieved by "smearing" the gluon field in the action in a particular way, ${ }^{3,4}$ and can be thought of as part of the standard Symanzik program for systematically removing discretization errors from lattice actions.

It is simple to "stagger" the naïve action and its improved variant to remove an exact degeneracy of a factor of 4 in tastes which arises from the spin degree of freedom. This results in an action with 4 doublers which can be simulated on the lattice using $\operatorname{det}(M)^{(1 / 4)}$ per flavor. It is very fast numerically because there is only one spin degree of freedom per site and the eigenvalues of $M$ are well behaved. This is what has allowed the MILC collaboration to generate ensembles of configurations which include $u, d$, and $s$ quarks in the vacuum with much more realistic masses than before. ${ }^{5}$

Some worries remain about potential nonlocality in the action as the result of taking the fourth root. However, this causes no problem in perturbative QCD where a simple power series in $x$ is obtained for an action with $\operatorname{det}(M)^{x}$. Stringent non-perturbative tests are also then needed. Luckily these tests are possible in this formalism with present day computers because of their speed, and are exactly the calculations required to test (lattice) QCD. The results, shown in the next section, speak for themselves.

\section{Recent Results}

The MILC collaboration have made sets of ensembles of gluon field configurations which include 2 degenerate light dynamical quarks $(u, d)$ and 1 heavier one $(s) .^{5}$ Taking the $u$ and $d$ masses as the same makes the lattice calculation much faster and leads to negligible errors in isospin-averaged quantities. The dynamical $s$ quark mass is chosen to be approximately correct based on earlier studies (in fact the subsequent analysis shows that it was slightly high and further ensembles are now being made with a lower value). The dynamical $u$ and $d$ quarks take a range of masses, down as low as a sixth of the (real) $m_{s}$. The sets of ensembles divide into two different values of the lattice spacing, $0.13 \mathrm{fm}$ and $0.09 \mathrm{fm}$, and the spatial lattice volume is $(2.5 \mathrm{fm})^{3}$ reasonably large. Analysis of hadronic quantities on these ensembles has been done by the MILC and HPQCD collaborations. $^{2}$

There are 5 bare parameters of QCD relevant to this analysis: $\alpha_{s}, m_{u / d}, m_{s}, m_{c}$ and $m_{b}$. The lattice 


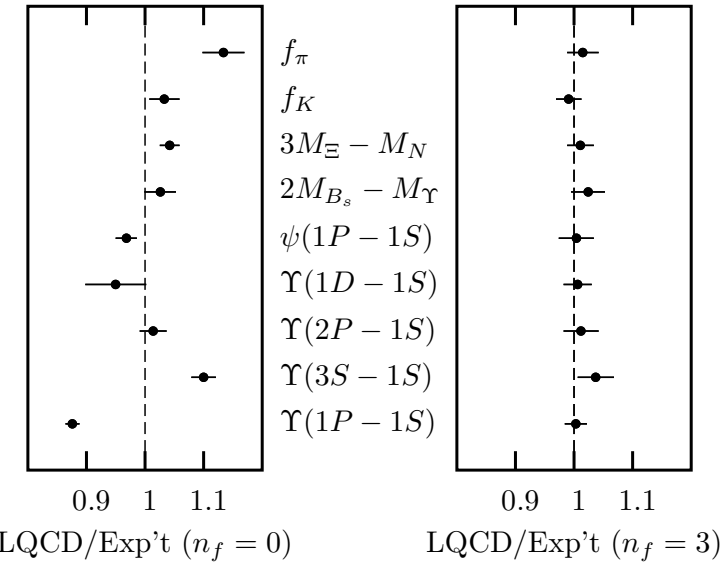

Figure 1. Lattice QCD results divided by experiment for a range of "gold-plated" quantities which cover the full range of hadronic physics. ${ }^{2}$ The unquenched calculations on the right show agreement with experiment across the board, whereas the quenched approximation on the left give systematic errors of $\mathcal{O}(10-20 \%)$.

spacing takes the place of $\alpha_{s}$ in lattice QCD. It is important that these parameters are fixed using the masses of "gold-plated" hadrons, i.e. hadrons which are well below their strong decay thresholds. Such hadrons are well-defined experimentally and theoretically and should be accurately calculable in lattice QCD. Using them to fix parameters will not then introduce unnecessary additional systematic errors into lattice results for other quantities. This has not always been done in past lattice calculations, particularly in the quenched approximation. It becomes an important issue when lattice QCD is to be used as a precision calculational tool. We use the radial excitation energy in the $\Upsilon$ system (i.e. the mass splitting between the $\Upsilon^{\prime}$ and the $\Upsilon$ ) to fix the lattice spacing and $m_{\pi}, m_{K}, m_{D_{s}}$ and $m_{\Upsilon}$ to fix the quark masses.

We can then focus on the calculation of other gold-plated masses and decay constants. If QCD is correct and lattice QCD is to work it must reproduce the experimental results for these quantities precisely. Figure 1 shows that this indeed works for the unquenched calculations with $u, d$ and $s$ quarks in the vacuum. A range of gold-plated hadrons are chosen which range from decay constants for light hadrons through heavy-light masses to heavyonium. This tests QCD in different regimes in which the sources of systematic error are very different and stresses the point that QCD predicts a huge range of physics with a small set of parameters.
References ${ }^{6-9}$ give more details on the quantities shown in Fig. 1. Here we will discuss some of these. Figure 2 shows the radial and orbital splittings in the $b \bar{b}(\Upsilon)$ system for the quenched approximation $\left(n_{f}=0\right)$ and with the dynamical MILC configurations with 3 flavors of dynamical quarks. Our physical understanding of the $\Upsilon$ system is very good and there are a lot of gold-plated states well below decay thresholds, which makes it a valuable system for lattice QCD tests. We use the standard lattice NRQCD effective theory for the valence $b$ quarks, which takes advantage of the non-relativistic nature of the bound states. The lattice NRQCD action is accurate through $v^{4}$ where $v$ is the velocity of the $b$ quark in its bound state. This means that spinindependent splittings, such as radial and orbital excitations, are simulated through next-to-leadingorder and should be accurate to $\approx 1 \%$. Thus the test of QCD using these splittings is a very accurate one. The fine structure in the spectrum is only correct through leading-order at present and more work must be done to bring this to the same level and allow tests against, for example, the splittings between the different $\chi_{b}$ states. $^{7}$

The $\Upsilon$ system is a good one for looking at the effects of dynamical quarks because we do not expect it to be very sensitive to dynamical quark masses. The momentum transfer inside an $\Upsilon$ is larger than any of the $u, d$ or $s$ masses and so we expect the radial and orbital splittings to simply "count" the number of dynamical quarks once we have reasonably light dynamical quark masses. The lower plot of Fig. 2 shows this to be true - the splittings are independent of the dynamical $u / d$ quark mass in the region we are working in (and therefore for the points plotted in the left-hand figure of Fig. 1 and in Fig. 2).

The $\pi$ and $K$ decay constants are important light hadron matrix elements, related to the purely leptonic decay rate via a $W$, and experimentally well-known. These are very sensitive to light quark masses and require a well-controlled extrapolation in the $u / d$ quark mass and interpolation in the $s$ quark mass to get accurate results to compare to experiment. Chiral perturbation theory can be used to perform the $u / d$ quark mass extrapolation provided the masses used on the lattice are small enough for the expansion in powers of quark mass $\left(\equiv m_{\pi}^{2} /\left(1 \mathrm{GeV}^{2}\right)\right)$ and its logarithms to work well. In practice this means that second-order chiral perturbation theory 


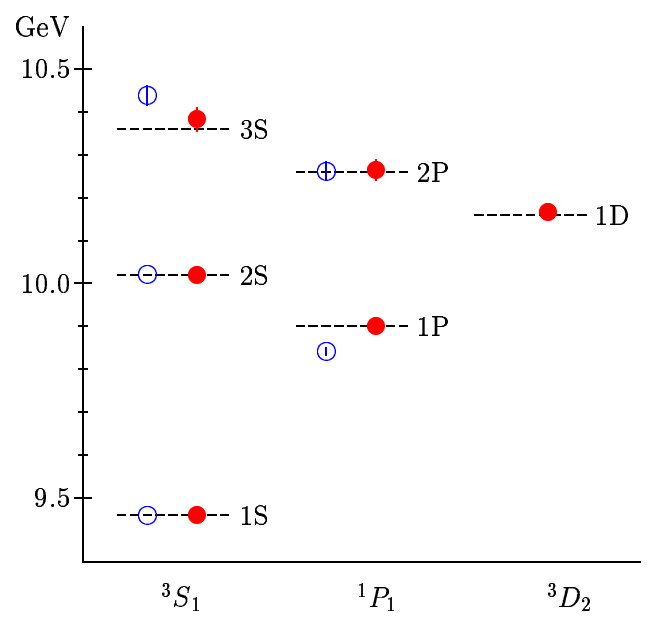

--- : Experiment $\bigcirc$ : Quenched MILC

- : $2+1$ flavors MILC, $m_{u, d}=m_{s} / 4, a=0.13 \mathrm{fm}$.

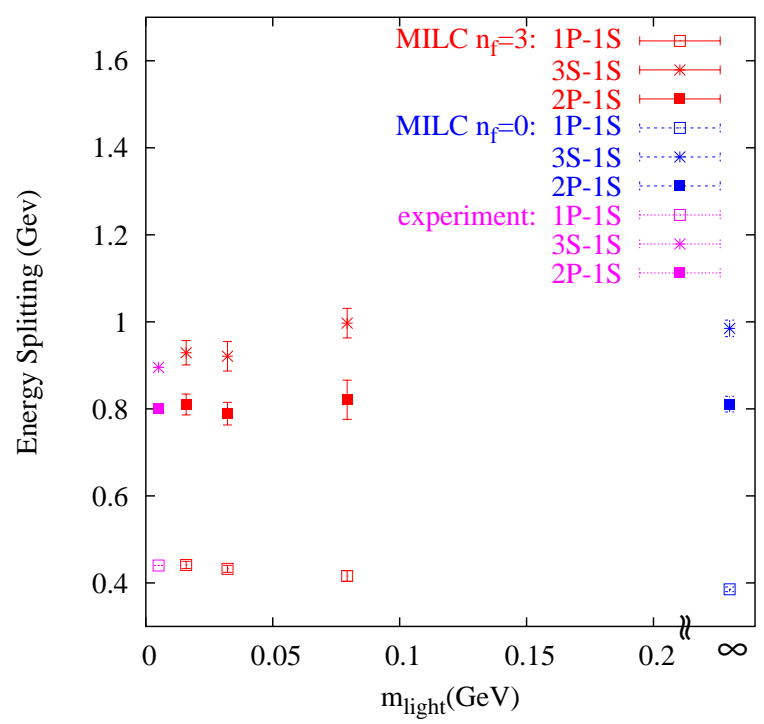

Figure 2. Radial and orbital splittings in the $\Upsilon$ system from lattice QCD in the quenched approximation and including $u, d$ and $s$ dynamical quarks. In this plot the lattice spacing was fixed from the radial excitation energy, i.e. the splitting between the $\Upsilon^{\prime}$ and the $\Upsilon$ and the $b$ quark mass was tuned to get the $\Upsilon$ mass correct. The bottom plot shows these splittings plotted as a function of the bare dynamical $u / d$ quark mass for several ensembles of MILC configurations. The leftmost lattice points are the ones used in the top plot and in Fig. 1.
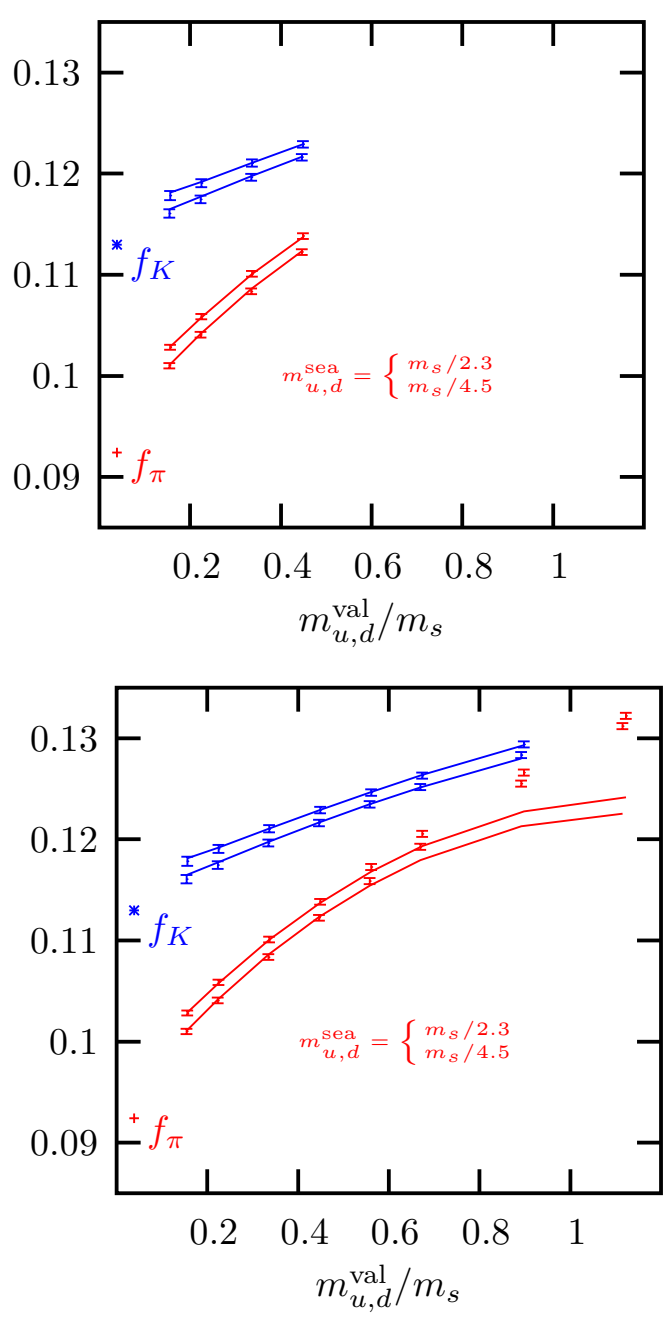

Figure 3. Results for the $\pi$ and $K$ decay constants as a function of the light quark mass for two dynamical MILC ensembles at a lattice spacing of $0.09 \mathrm{fm}$. The top plot shows the chiral extrapolation using only results with valence $u / d$ quark masses $<m_{s} / 2 .^{2}$ The chiral extrapolation must subsequently be corrected for the incorrect valence and sea $s$ quark mass to give the results in Fig. 1. The bottom plot shows that this chiral fit from light $u / d$ quark masses does not agree well with the data for $m_{u / d}>m_{s} / 2$. 
should work at the $2 \%$ level for $m_{u / d}<m_{s} / 2$. Note that the error is set by the largest quark mass used in the chiral fits, not the smallest.

Figure 3 shows the results and chiral extrapolation for the decay constants on the ensembles of MILC configurations with $m_{u / d}^{s e a}=m_{s} / 2.3$ and $m_{s} / 4.5$ at a lattice spacing of $0.09 \mathrm{fm}$. The curves in the top plot show the chiral extrapolation using only results with $m_{u / d}^{\text {valence }}<m_{s} / 2$. This extrapolation has to be corrected, using the lattice results, to interpolate to the physical $s$ quark mass for both sea and valence $s$ quarks. This then gives the results shown in Fig. 1 which agree with experiment. The lower plot shows what happens when the chiral extrapolation fit obtained in the top plot is evaluated for larger valence $m_{u / d}$. The $f_{\pi}$ results start to show clear disagreement for $m_{u / d}>m_{s} / 2$, which makes the problem of performing accurate chiral extrapolations using results with $m_{u / d}>m_{s} / 2$ obvious. Previous lattice calculations have been forced by computing cost to work only in this regime, with the added problem that the sea $m_{u / d}$ is also large. ${ }^{10}$

Another gold-plated hadron mass is that of the nucleon. A full chiral extrapolation of the results for this on the MILC configurations has not yet been done. The upper plot of Fig. 4 shows very encouraging signs that an answer in agreement with experiment will be found. ${ }^{6}$ There is a clear sign of dependence on the lattice spacing, however, which will have to be taken into account. Combinations of baryon masses can be made which are relatively insensitive to $u / d$ quark masses and other effects, and it is one of these, $3 m_{\Xi}-m_{N}$, which is plotted in Fig. 1.

It is important to realize that accurate lattice QCD results are not going to be obtainable in the near future for every hadronic quantity of interest. What these results show is that "gold-plated" quantities should now work. Gold-plated hadrons are those well below decay threshold for strong decays. Unstable hadrons, or even those within $100 \mathrm{MeV}$ or so of Zweig-allowed decay modes, have a strong coupling to their real or virtual decay channel which is not correctly simulated on the lattice. The problem is that, with the lattice volumes being used, the allowed nonzero momenta are typically greater than $400 \mathrm{MeV}$ and this significantly distorts the decay channel contribution. Much larger simulations will be necessary to handle these hadrons.
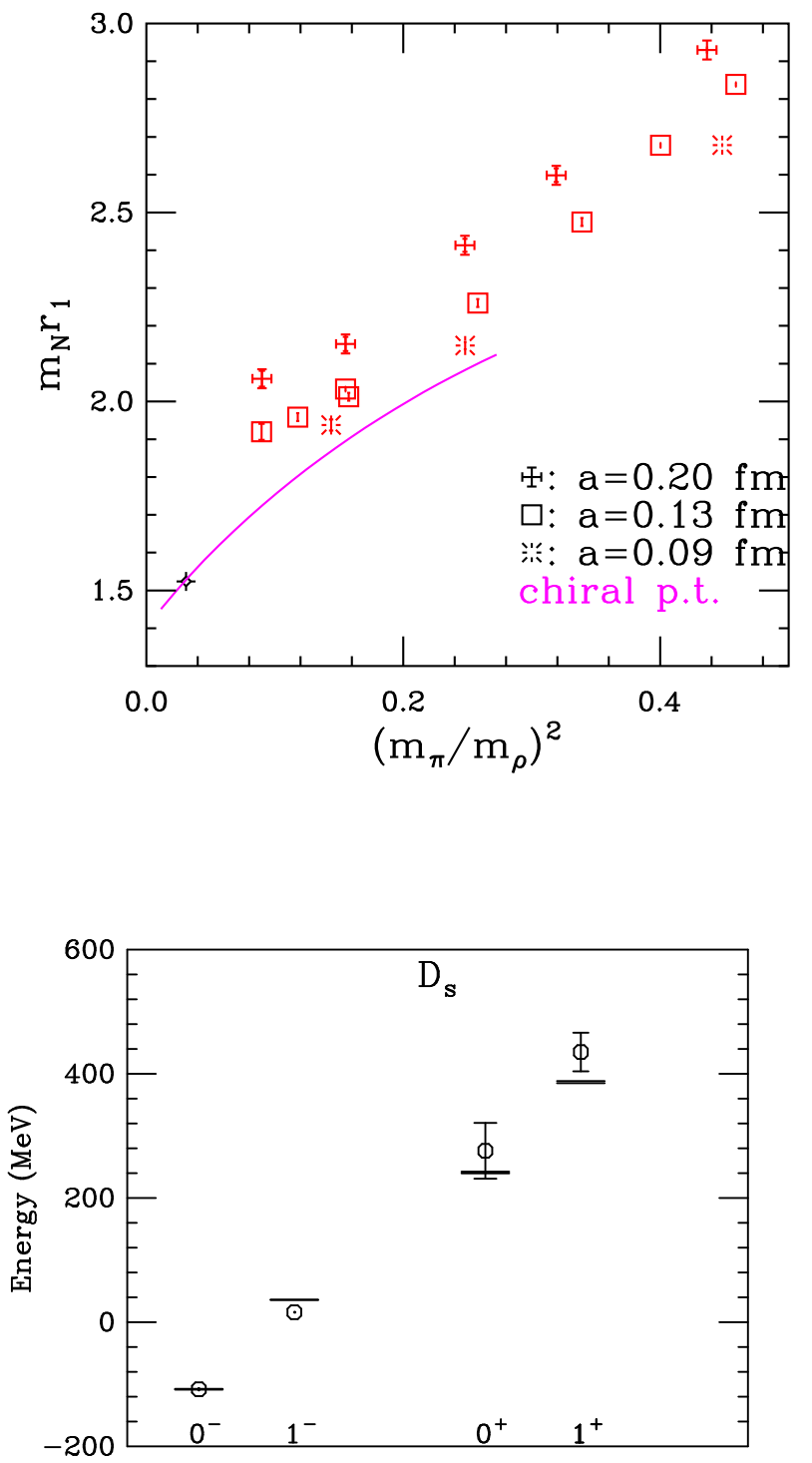

Figure 4. The top plot shows results for the nucleon mass on MILC ensembles for different lattice spacings and dynamical quark masses. The nucleon mass is given in units of $r_{1}$, a parameter from the heavy quark potential whose physical value is $0.32 \mathrm{fm}$. The dynamical quark mass is indicated by the variable $m_{\pi}^{2} / m_{\rho}^{2}$. The curve roughly indicates chiral perturbation theory. ${ }^{6}$ The bottom plot shows the spectrum of $D_{s}$ states obtained from the MILC dynamical configurations with $m_{u / d}=m_{s} / 4$ and lattice spacing $0.13 \mathrm{fm} .{ }^{11}$ 
Gold-plated hadrons include: $\pi, K, D, D_{s}, J / \psi$, $\Upsilon, B, B_{s}, p, n, \Lambda, \Omega$, etc.. The following are not goldplated: $\rho, \phi, D^{*}, D_{s J}, \Delta, N^{*}$, pentaquarks, glueballs and hybrids in general. Lattice calculations will not get the masses right for non-gold-plated hadrons even when light dynamical quarks are included. This does not preclude lattice calculations giving useful qualitative results and insight but these points should be borne in mind for any quantitative comparison.

Figure 4 also shows the spectrum of $D_{s}$ states obtained on the dynamical MILC configurations. ${ }^{11}$ The valence $c$ quarks are simulated using an effective theory which, in a similar way to the $\Upsilon$ above, should be accurate for spin-independent splittings and not quite so accurate for fine structure in the spectrum. The hyperfine splitting between the $D_{s}$ and $D_{s}^{*}$, for example, is currently missing a radiative correction to the term in the action proportional to the spin coupling to the chromo-magnetic field. This is being calculated in lattice perturbation theory. ${ }^{1}$ Also shown are the scalar and axial vector orbital excitations compared to the recent experimental results for these mesons. The lattice calculation is giving a high result, albeit with large statistical errors at present. However, a high result is consistent with the fact that these mesons are not gold-plated and the lattice calculation does not currently include correctly the coupling to their decay modes.

Decay rates which can be accurately calculated for gold-plated hadrons are those in which there is at most one (gold-plated) hadron in the final state. This therefore includes leptonic and semi-leptonic decays and the mixing of neutral $B$ and $K$ mesons. Luckily there is a gold-plated decay mode available to extract each element (except $V_{t b}$ ) of the CKM matrix which mixes quark flavors under the weak interactions in the Standard Model:

$$
\left(\begin{array}{ccc}
\mathbf{V}_{\mathbf{u d}} & \mathbf{V}_{\mathbf{u s}} & \mathbf{V}_{\mathbf{u b}} \\
\pi \rightarrow l \nu & K \rightarrow l \nu & B \rightarrow \pi l \nu \\
& K \rightarrow \pi l \nu & \\
\mathbf{V}_{\mathbf{c d}} & \mathbf{V}_{\mathbf{c s}} & \mathbf{V}_{\mathbf{c b}} \\
D \rightarrow l \nu & D_{s} \rightarrow l \nu & B \rightarrow D l \nu \\
D \rightarrow \pi l \nu & D \rightarrow K l \nu & \\
\mathbf{V}_{\mathbf{t d}} & \mathbf{V}_{\mathbf{t s}} & \mathbf{V}_{\mathbf{t b}} \\
\left\langle B_{d} \mid \bar{B}_{d}\right\rangle & \left\langle B_{s} \mid \bar{B}_{s}\right\rangle &
\end{array}\right)
$$

As described earlier, the determination of the CKM elements and tests of the self-consistency of the CKM matrix are the current focus for the search for beyond

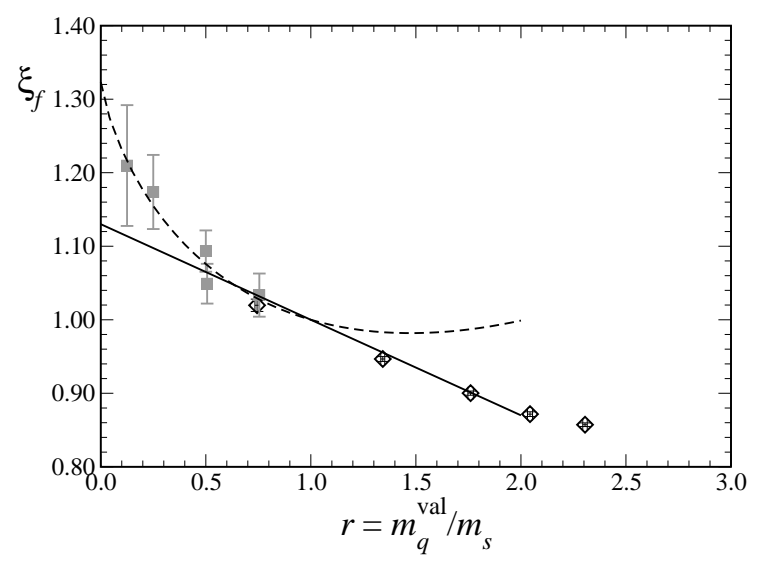

Figure 5. Results for the ratio of $f_{B_{s}} / f_{B_{d}}$, as a function of valence $u / d$ quark mass in units of $m_{s} .{ }^{12}$ The (grey) squares are from the dynamical MILC ensembles including $u, d$ and $s$ dynamical quarks. ${ }^{13}$ The (black) diamonds are from the previous best calculation which included 2 flavors of dynamical quarks with masses $>m_{s} / 2 \cdot{ }^{10}$ The straight line is a linear extrapolation for the 2 flavor results, the curve includes the possibility of logarithms from chiral perturbation theory. This ratio, for physical $u / d$ masses, appears in the ratio of oscillation frequencies for $B_{s}$ and $B_{d}$ mesons, which it is hoped to measure experimentally.

the Standard Model physics and lattice calculations of these decay rates will be a key factor in the precision with which this can be done.

The first calculations on the dynamical MILC configurations have concentrated on the $B$ and $B_{s}$ leptonic decay rates, ${ }^{13,11}$ because these are the simplest. They are parameterized by the decay constants, $f_{B}$ and $f_{B_{s}}$, and these are an important component of the mixing rate for these mesons, which constrains $V_{t s}$ and $V_{t d}$. Again one issue in extracting reliable lattice results for $f_{B}$ and $f_{B_{s}}$ is the chiral extrapolation in the $u / d$ quark mass. Figure 5 shows results on the MILC configurations for the ratio of $f_{B_{s}} / f_{B_{d}}$ plotted against the valence $u / d$ quark mass. ${ }^{12}$ The data extend into the region $m_{u / d}<$ $m_{s} / 2$ which will allow an accurate chiral extrapolation for the first time. Although the statistical errors are currently rather large, it seems likely that the result for this ratio will be larger than previous estimates based on extrapolations from larger $u / d$ masses, and including only two flavors of dynamical quarks. ${ }^{10}$ Further calculations of gold-plated matrix elements are in progress. ${ }^{14}$ 


\section{Conclusions}

The possibility of few percent errors in lattice QCD simulations creates a superb opportunity for lattice QCD to have an impact on particle physics. Lattice QCD is essential to the high precision $B / D$ physics programs at BaBar, Belle, CLEO-c, Fermilab,.... Predicting BaBar/Belle, and especially CLEO-c results at the few percent level will give much needed credibility to lattice QCD. It is critical in such tests and applications to focus on gold-plated quantities.

High precision non-perturbative QCD is a landmark in the history of quantum field theory, and it is an essential first step in our preparation for strongcoupling beyond the Standard Model.

\section{Acknowledgments}

We are grateful to PPARC, NSF, DoE and the EU for funding this work, and to all our collaborators ${ }^{2}$ for many useful discussions. A version of this talk was also presented at the Hadron 2003 Conference and is published in the proceedings of that meeting.

\section{References}

1. H. Trottier, Proceedings of LAT03, hep-lat/0310044.

2. C. Davies et al., MILC/HPQCD/FNAL/UKQCD collaborations, hep-lat/0304004.

3. G. P. Lepage, Phys. Rev. D 59, 074502 (1999), heplat/9809157.

4. K.Orginos, D. Toussaint and R. L. Sugar, Phys. Rev. D 60, 054503 (1999), hep-lat/9903032.

5. C. Bernard et al., Phys. Rev. D 64, 054506 (2001), hep-lat/0104002.

6. S. Gottlieb, Proceedings of LAT03, hep-lat/0310041.

7. A. Gray et al., Nucl. Phys. B (Proc. Suppl. 119), 592 (2003), hep-lat/0209022; C. Davies et al., ibid 595, hep-lat/0209122.

8. M. di Pierro et al., Proceedings of LAT03, heplat/0310042.

9. C. Aubin et al., Proceedings of LAT03, heplat/0309088.

10. S. Aoki et al., hep-ph/0307039.

11. M. di Pierro et al., Proceedings of LAT03, heplat/0310045.

12. A. Kronfeld, Proceedings of LAT03, hep-lat/0310063.

13. M. Wingate, Proceedings of LAT03, hep-lat/0309092.

14. J. Shigemitsu et al., Proceedings of LAT03, heplat/0309039; M. Okamato et al., Proceedings of LAT03, hep-lat/0309107. 


\section{DISCUSSION}

Jeff Appel (Fermilab): Congratulations on your progress on masses and engineering numbers needed for CKM measurements. The major issues in QCD itself, however, lie in the area you described as still hard after current successes: e.g. light scalar mesons, glueballs, hybrids, and production processes. When might we expect progress in these areas? Are the new improvements enough or are yet newer techniques needed?

Peter Lepage: It's difficult. You can do some resonance work. There are techniques for lowmass resonances so things like that $\rho$ and the $\phi$ can probably be nailed - we have the technology. Things like glueballs - which would be really neat - are much, much harder and people have really just scratched the surface in thinking about new ways of doing it. So I don't know what to promise there. The one thing I can say, though, about things like glueballs is that in lattice gauge theory, we really haven't been sure that it was working for anything up until very, very recently. And when you're not sure that it's working for anything, even simple things, it's really, really hard to devote a lot of time to something like the glueball which you know is much much harder, given that you're not even clear that it can get the nucleon mass right yet. And, so at some level, getting a foundation that is really, really solid and well-established - at the couple of percent level where everyone agrees that this is the real thing - is bound to improve the odds for doing something non-trivial like figuring out how to do a glueball decay width. I can't predict what it is, I'm just saying that we're in vastly better shape by virtue of having gotten to first base. We have a much better chance of getting to second base if we've already made it to first base. And what we're doing now is trying to get to first base, and looking for ways in which we can have a big impact. And that's why there's a lot of focus on $B$ physics, because there we really can have a big impact on the scientific program. This is not to say that there's nothing you can get from lattice gauge theory. I mean, probably the most compelling studies so far for things like glueballs come from lattice gauge theory. And people have fooled around with techniques for trying to estimate decay widths and so on - even of glueballs. I don't know if those techniques are reliable or not, but there are some ideas that you can try out. And if we can sort of pin down the rest of the territory then it makes it more likely that we'll be able to figure our way out of that problem. So I'm not being too encouraging, but I think we're in much better shape even for the harder problems.

Enrico Predazzi (INFN, Turin): When you mentioned in the beginning that the old lattice did not really represent $\mathrm{QCD}$, you mentioned that that was, among other things, because of not incorporating quark loops. Now, in the new lattice, you have sea quarks but you have no actual gluons or anything of the kind.

Peter Lepage: No, we have gluons.

Enrico Predazzi (INFN, Turin): How does that come in?

Peter Lepage: Basically, we're evaluating the path integral of QCD numerically so we're integrating over all values of the gluon field. I should be clear for people who don't know lattice QCD. What it is is taking the path integral of the quantum field theory - literally the thing you read in a textbook - and evaluating that integral numerically. It's a numerical approximation to that integral. It's a very hard integral to do, and it's taken us a while to figure out how to do it effectively, but it is just the path integral that's the input. That's what the computer program is munching on. When I say bare quark mass and bare coupling as the inputs, those are literally the things that appear in the Lagrangian. We're working with the real thing here. I showed you upsilon spectra - it's not a quark model with a potential - there are only 5 numbers that went into all the data I showed you, and those are those masses and the bare thing and the rest of it is a numerical path integral. 
Alberto Sirlin (New York University): One calculation that would be very interesting for the problem of universality is the calculation of $f^{+}(0)$ in the $K_{\ell 3}$ decays - the form factor. It seems to fall into the category of your goldplated...could that be possible? That would be very important.
Peter Lepage: Yes. There's a big experimental community in $B$ and $D$ physics, but we know that there's also some really interesting stuff in kaon physics. So we're aware of it. Whether it gets done this year or next year is another question. It might actually be useful to talk a little bit - maybe you should visit Cornell? 